\title{
ELEMENTOS TRAÇO EM SEDIMENTOS URBANOS E SUA AVALIAÇÃO POR GUIDELINES
}

\section{TRACE ELEMENTS IN URBAN SEDIMENTS AND THEIR GUIDELINES EVALUATION}

\section{Cristiano Poleto'; Gustavo Henrique Merten ${ }^{1}$}

\author{
${ }^{1}$ IPH - UFRGS. Av. Bento Gonçalves, 9500, 91501-970, Porto Alegre, RS, Brasil.
}

\section{RESUMO}

Os recursos hídricos em bacias urbanas têm sido severamente prejudicados não só pelas alterações hidrológicas, mas especialmente pela carga poluente que é transferida da bacia vertente para os corpos d'água. Por isto, o presente trabalho pretende apresentar o resultado de análises de digestão ácida de sedimentos fluviais em suspensão em uma bacia urbana, estabelecer comparações com valores de referência (Guidelines) e com os valores de background local. A área de estudo é uma bacia urbana residencial, localizada na região metropolitana de Porto Alegre e com aproximadamente $0,83 \mathrm{~km}^{2}$. As amostras de sedimentos fluviais em suspensão foram coletadas entre os anos de 2003 e 2006. Foram realizadas digestões ácidas dos sedimentos com $\mathrm{HCl}-\mathrm{HF}-\mathrm{HClO}_{4}-\mathrm{HNO}_{3}$ para obtenção das concentrações totais de $\mathrm{Co}, \mathrm{Cd}$ e As. Os resultados obtidos nas digestões ácidas dos sedimentos fluviais em suspensão possibilitaram verificar a variabilidade entre os Guidelines. Os valores de background local possibilitaram verificar a existência do enriquecimento da área de estudo por As e metais como o Co e o Cd.

Palavras-chave: Guidelines. Background. Sedimentos fluviais em suspensão. Metais, Bacia urbana.

\section{ABSTRACT}

Water resources have been severely damaged by hydrologic alterations and especially by pollutants transferred from the urban watershed. This paper presents results of total acid analysis of suspended sediments in an urban watershed and establishes comparisons with three different sets of Guidelines and local background results. The watershed studied has an area of approximately $0.83 \mathrm{~km}^{2}$, and is located in the Porto Alegre metropolitan area, the largest city in southern Brazil. Suspended 
sediment samples were collected in the watershed outlet from 2003 to 2006. Total acid digestion analyses $\left(\mathrm{HCl}-\mathrm{HF}-\mathrm{HClO}_{4}-\mathrm{HNO}_{3}\right)$ were performed to obtain the total concentrations of $\mathrm{Co}, \mathrm{Cd}$ and As. The results of the acid analysis of the suspended sediments and the background values demonstrated the existence of an enrichment process due to the presence of pollutants in the environment, such as the metals $\mathrm{Co}$ and $\mathrm{Cd}$, and the variability of the Guidelines.

Keywords: Guidelines. Background. Suspended sediments. Metals. Urban watershed.

\section{INTRODUÇÃO}

O desenvolvimento urbano desordenado pode causar várias alterações tanto no ecossistema terrestre quanto no ecossistema aquático. Dentre as ações antrópicas de maior impacto, pode-se citar a substituição da vegetação original por áreas impermeáveis (PACKMAN et al., 1999) que aumentam o escoamento superficial e diminuem a taxa de infiltração de água, a concentração e o lançamento de grandes cargas de esgoto in natura e a adição de contaminantes químicos através das mais diversas fontes.

Segundo Patkowska, Hursthouse e Kij (2005), a dinâmica natural das interações antrópicas com o uso do solo como um espaço urbano, freqüentemente resulta na introdução de uma ampla variedade de materiais que apresentam uma grande diversidade nas suas características físico-química, e por isso o ambiente urbano torna-se tão complexo. Parte desses materiais introduzidos nas áreas urbanas pode ser caracterizada como poluentes e poderão atingir os corpos d'água durante os períodos chuvosos e, dependendo do tipo de substância e de suas concentrações, poderão dar início a alterações em todo o ecossistema aquático. O gerenciamento sustentável do ambiente urbano requer, então, a identificação dessas alterações para possibilitar a remediação dos danos causados ao ambiente.

As alterações que ocorrem no ecossistema aquático refletem em parte os impactos sofridos pelo ecossistema terrestre, sendo os sedimentos um importante elo entre esses dois sistemas. Neste contexto, os estudos voltados para a caracterização da qualidade dos sedimentos podem se constituir em um bom indicador das alterações sofridas nessas áreas, uma vez que estudos ao redor do mundo têm apontado um rápido crescimento nas concentrações de contaminantes nos sedimentos a partir da industrialização e/ou urbanização de determinadas áreas (TAYLOR, BIRCH e LINKS, 2004).

As alterações impostas pelas ações antrópicas nas áreas urbanas, além de implicações aos aspectos hidrológicos, provocam também sérios problemas de qualidade da água, seja esta superficial ou subsuperficial. Isso ocorre principalmente devido aos dejetos domiciliares ou industriais, líquidos ou sólidos, que são lançados diretamente nos corpos d'água ou que se infiltram através do solo. Para Deletic 
(2001), as fontes não-pontuais de poluentes são consideradas como as principais causas de alteração da qualidade de água dos cursos d'água em áreas urbanas. Os impactos negativos à qualidade da água são causados principalmente pela presença dos sedimentos que cada vez mais, vêm sendo considerados como um poluente importante para os ecossistemas fluviais devido ao impacto negativo que podem causar não só à qualidade da água, mas também à biota aquática (RUSSELL, WALLING e HODGKINSON, 2001). Segundo Pardos et al. (2004), os sedimentos contaminados são considerados mundialmente como os contaminantes que mais contribuem para a degradação do ecossistema aquático e, portanto, é um problema eminente a ser resolvido para a preservação dos recursos hídricos.

Assim, o presente trabalho tem o objetivo de apresentar os estudos realizados com sedimentos coletados em uma bacia urbana e avaliar os resultados das concentrações totais de três elementos presentes nessas amostras. Os três elementos foram propositadamente selecionados para demonstrar que de uma forma geral a utilização dos valores estabelecidos por diferentes Guidelines, para avaliações ambientais, apresentam grandes limitações.

\section{REVISÃO BIBLIOGRÁFICA}

Tipos de poluentes encontrados em áreas urbanas

Os poluentes em áreas urbanas variam muito, desde compostos orgânicos a metais altamente tóxicos. Três grupos de contaminantes são freqüentemente identificados em sedimentos aquáticos urbanos e por isso pertencem aos grupos que formam os Guidelines, ou valores de referência, para os sedimentos conforme a sua toxicidade sobre a biota aquática: elementos traço, hidrocarbonetos e compostos organoclorados (METRE e MAHLER, 2003).

Os elementos traço, especialmente os chamados "metais pesados", estão entre os poluentes mais freqüentemente encontrados no ambiente, e sua ocorrência em águas e biota indica a presença de fontes que podem ser naturais e/ou antropogênicas (FOSTER e CHARLESWORTH, 1996; SINGH et al., 2005). Segundo Baird (2002), estes elementos possuem densidades altas em comparação com outros materiais comuns, muitos deles representam riscos à saúde humana e sua fixação final ocorre em solos e sedimentos. Em contraste com os poluentes orgânicos, os metais pesados não são biologicamente ou quimicamente degradados, e assim poderão tanto se acumular localmente como serem transportados por longas distâncias (MARCHAND et al., 2006).

Para Porto (1995), os metais pesados, como $\mathrm{Cd}, \mathrm{Cu}, \mathrm{Cr}, \mathrm{Pb}, \mathrm{Hg}, \mathrm{Ni}, \mathrm{Zn}$ e, ainda, As, Co e Se, são um grupo de elementos químicos que aparece com bastante freqüência em áreas urbanizadas. Segundo Banerjee (2003), $\mathrm{Pb}$ e $\mathrm{Cd}$ são dois elementos freqüentemente estudados em sedimentos urbanos, mas muita atenção deve ser dada também a outros elementos traço, tais como $\mathrm{Cr}, \mathrm{Cu}, \mathrm{Zn}$ e $\mathrm{Ni}$, que 
também são normalmente encontrados em ambientes urbanos. Para Gromaire et al. (2001), os metais mais impactantes e comuns em áreas urbanas são $\mathrm{Cd}, \mathrm{Cu}, \mathrm{Pb}$ e $\mathrm{Zn}$. Para Horowitz (1995), o próprio sedimento é um poluente, uma vez que muitos elementos traço, tal qual $\mathrm{Cu}, \mathrm{Zn}, \mathrm{Cd}, \mathrm{Cr}, \mathrm{Pb}$ e $\mathrm{Ni}$, freqüentemente detectados em escoamentos de pavimentos urbanos, estão associados a esses sedimentos.

O As é um semimetal que também é comumente encontrada em áreas urbanas, e a sua toxicidade depende principalmente de sua forma molecular e do seu estado de oxidação (SÁNCHEZ-RODAS et al., 2005). O As possui uma maior tendência para formar ligações iônicas, ao invés de covalentes, devido a seu maior caráter metálico (BAIRD, 2002).

De uma forma geral, algumas substâncias são normalmente utilizadas para diferentes funções no ambiente urbano, tais como inseticidas e fertilizantes, tornandose posteriormente poluentes para esse mesmo meio. Em outras situações, o poluente pode ser um subproduto derivado de outras substâncias. Este é o caso do $\mathrm{Pb}$, que é proveniente das emissões de automóveis (queima de combustível) e óleos originados de vazamentos de veículos automotores. Os materiais particulados que se depositam nas ruas e rodovias, geralmente chamados de poeira ou sedimentos de ruas, são poluentes significantes nas áreas urbanas porque eles contêm altos níveis de metais tóxicos e contaminantes orgânicos, tais como hidrocarbonetos policíclicos aromáticos (HOPKE, LAMB e NATUSCH, 1980; e FERGUSSON e KIM, 1991; DE MIGUEL et al., 1997; LI, POON e LIU., 2001). Todos esses poluentes e a fuligem resultante das emissões de gases dos veículos, das indústrias e da queima de resíduos, se depositam nas superfícies impermeabilizadas e posteriormente são lavados pela chuva e, assim, a água resultante desta lavagem chega aos rios contaminando-os.

Guidelines para a avaliação da qualidade de sedimentos

Em estudos de qualidade ambiental torna-se interessante avaliar as concentrações de metais com respeito a valores de referência ou Guidelines, o que possibilita caracterizar a extensão da poluição e seus possíveis impactos ecológicos. Segundo Canadian Council of Ministers of The Environment (CCME, 1995), os Guidelines são limites numéricos ou recomendações narrativas para dar suporte ou conservar designados usos do meio ambiente aquático. De acordo Ontario Ministry of the Environment and Energy (OMEE, 1993), o propósito dos Guidelines de qualidade de sedimentos é proteger o ambiente aquático, propondo níveis seguros para concentrações de metais, nutrientes (substâncias na qual promovem o crescimento do fitoplancton) e compostos orgânicos presentes nesses sedimentos.

Historicamente devido à necessidade de orientar o destino ou gerenciar materiais originados de dragagens, inúmeras agências internacionais desenvolveram ou estão desenvolvendo critérios para a qualidade dos sedimentos. O primeiro Guideline em sedimentos foi desenvolvido pela Federal Water Quality Administration e adotado pela United States Environmental Protection Agency (U.S.E.P.A.) em 1973. Os critérios incluíam sete parâmetros de avaliação e se algum 
valor numérico fosse excedido, o sedimento era classificado como poluído e deveria ser tratado.

Mais recentemente, o Ontario Ministry of the Environment and Energy (OMEE, 1993) desenvolveu um Guideline com três níveis de efeitos ecotoxicológicos, sendo esses baseados em efeitos crônicos e de longo prazo sobre os organismos bênticos. As concentrações de alguns elementos fixadas por essa agência podem ser observadas na Tabela 1. Esse Guideline recomenda limites para contaminantes individuais e a preocupação do seu desenvolvimento foi baseada nos efeitos dessas substâncias na biota associada aos sedimentos (organismos bentônicos), o que é considerado o ideal por Lijzen et al. (2001).

TABELA 1 - Guideline de qualidade de sedimentos do Ontario Ministry of the Environment and Energy.- OMEE para nutrientes, metais, e compostos orgânicos (concentração total em $\mu \mathrm{g} \mathrm{g}^{-1}$ de peso seco)

\begin{tabular}{ccc}
\hline Elementos & Nível de efeito: $\mathbf{b a i x o}\left(\mu \mathbf{g ~ g}^{\mathbf{- 1}}\right)$ & Nível de efeito: $\mathbf{s e v e r o}\left(\mu \mathbf{g} \mathbf{g}^{\mathbf{- 1}}\right)$ \\
\hline Arsênio $(\mathrm{As})$ & 6 & 33 \\
Cádmio $(\mathrm{Cd})$ & 0,6 & 10 \\
Cobalto $(\mathrm{Co})$ & - & 50 \\
\hline
\end{tabular}

Adaptado de: OMEE (1993)

Para se formular esses critérios ou limites são realizadas comparações entre sedimentos contaminados de uma determinada localização e seus respectivos backgrounds, que são considerados como o material de referência local, uma vez que os níveis de contaminação são aceitáveis devido à presença de organismos indicadores, ou seja, organismos adaptados a essas concentrações naturais (MUDROCH; AZCUE, 1995).

$\mathrm{Na}$ Holanda, que tem uma longa história de políticas de proteção de solos e sedimentos datada desde 1962, os Guidelines foram reformulados em meados dos anos 90, introduzindo métodos ecotoxicológicos e considerando também o potencial humano de exposição aos contaminantes (BIRD et al., 2003; LEE et al., 2005).

Os critérios estabelecidos pelo Guideline holandês (Tabela 2) têm sido adotados por muitos países da Europa e usados em numerosos estudos de poluição por metais em diversos países como meio de avaliação dos níveis de contaminação (MACKLIN; KLIMEK, 1992; MACKLIN et al., 2003; BIRD et al., 2003; LEE et al., 2005). Isso porque o Guideline holandês foi empregado em muitas bacias e estudos, se estabelecendo como valores que foram amplamente testados (BIRD et al., 2003). 
TABELA 2 - Guideline Holandês para concentrações totais de metais em sedimentos, com valores de referência limite e de intervenção.

\begin{tabular}{ccc}
\hline Elementos & Concentração limite $\left(\mu \mathbf{g ~ g}^{\mathbf{- 1}}\right)$ & Concentração de intervenção $\left(\mu \mathbf{g ~ g}^{\mathbf{- 1}}\right)$ \\
\hline Arsênio (As) & 29 & 55 \\
Cádmio (Cd) & 0,8 & 12 \\
Cobalto (Co) & 9 & 240 \\
\hline
\end{tabular}

Adaptado: Ministery of Housing Spatial Planning And Environment - VROM (2000)

O Brasil através do Ministério do Meio Ambiente editou uma resolução para materiais dragados em águas jurisdicionais brasileiras, a Resolução do Conselho Nacional do Meio Ambiente -CONAMA n 344 de 25 de março de 2004, visando estabelecer diretrizes gerais e procedimentos mínimos para a avaliação do material a ser dragado e o gerenciamento da sua disposição em terra (BRASIL, 2004). Assim, foram fixados dois níveis ou limites para o material a ser dragado:

- Nível I: limiar abaixo do qual prevê-se baixa probabilidade de efeitos adversos à biota;

- Nível II: limiar acima do qual prevê-se um provável efeito adverso à biota.

De uma forma geral, como a resolução se baseou em referências internacionais, como as canadenses e americanas, esta acaba reproduzindo os mesmos conceitos e recomendações, como por exemplo a adoção dos valores de background local, ou seja, obtidos na área de estudo. Os valores de referência dessa Resolução para alguns elementos são apresentados na Tabela 3.

TABELA 3 - Valores de referência estabelecidos pela Resolução CONAMA nº 344/2004

\begin{tabular}{|c|c|c|}
\hline \multirow[t]{2}{*}{ Elementos } & \multicolumn{2}{|c|}{$\begin{array}{l}\text { Níveis de classificação do material a ser dragado } \\
\text { (em unidade do material seco) }\end{array}$} \\
\hline & Nível I $\left(\mu \mathrm{g} \mathrm{g}^{-1}\right)$ & Nível II $\left(\mu \mathrm{g} \mathrm{g}^{-1}\right)$ \\
\hline Arsênio (As) & 5,9 & 17 \\
\hline Cádmio (Cd) & 0,6 & 3,5 \\
\hline
\end{tabular}

Adaptado de: Brasil (2004)

Apesar de sua grande utilidade em estabelecer parâmetros para se avaliar a qualidade dos sedimentos, todos os Guidelines possuem várias limitações. Segundo Mudroch e Azcue (1995), a maior objeção imposta aos Guidelines é a sua falta de universalização, pois estes são completamente específicos ao local de sua criação. Para Poleto e Gonçalves (2006), a especificidade de cada valor de referência fica nítida ao se comparar os valores limites estabelecidos pelo Guideline Holandês com os propostos pelo Canadense. Os valores encontrados para estudos na Holanda são muito altos em comparação aos do Canadá, isso devido, principalmente, às diferenças 
mineralógicas de cada região, e possibilitando um bom exemplo de como é extremamente arriscado utilizar-se de estudos produzidos em outras regiões ou países.

Para melhorar a avaliação dos níveis de metais encontrados nas áreas de estudo é muito importante a utilização de valores de background locais. Estes podem ser obtidos através de amostras compostas coletadas em áreas vegetadas sem alterações antrópicas ou do fundo de lagos, ou seja, áreas que não foram afetadas por descargas de contaminantes e, portanto, preservam as características originais do local (OMEE, 1993). As concentrações totais de metais contidas nesses sedimentos possibilitam uma informação importante sobre os níveis originais (BUYKX et al., 2000). A utilização de concentrações dos backgrounds pode ser útil tanto para comparações entre os resultados de estudos em áreas degradadas, quanto para a tomada de decisões quanto a remediação de áreas contaminadas ou potencialmente contaminadas. Para Hursthouse (2001), o não reconhecimento dos valores de background local poderá sub ou, superestimar a significância dos níveis de contaminação. Entre as desvantagens na utilização apenas dos valores do background está a falta de parâmetros ecotoxicológicos que poderão afetar os organismos (OMEE, 1993).

\section{MATERIAIS E MÉTODOS}

Caracterização da área de estudo

A sub-bacia urbana em estudo está inserida na Vila Santa Isabel, localizada no município de Viamão, região metropolitana de Porto Alegre, capital do estado do Rio Grande do Sul, no sul do Brasil. A área de estudo possui aproximadamente $0,83 \mathrm{~km}^{2}$.

\section{Levantamentos sobre infra-estrutura básica}

O levantamento da infra-estrutura básica existente foi realizado com a aplicação de um questionário onde foram entrevistadas 659 casas da bacia em estudo (de um total de 1.733 casas, portanto a amostra foi de 38,03\%) e com o levantamento de campo que contou com o auxílio de Sistema de Posicionamento Global - GPS e fotografias digitais. Os resultados obtidos formaram um banco de dados que foi inserido na imagem de satélite através do software $\operatorname{ArcView}^{\circledR}$ e geraram mapas com as distribuições dessas informações.

\section{Coleta de amostras para o "background"}

Para a obtenção das concentrações de Co, Cd e As, que foram utilizadas como background da bacia hidrográfica, realizaram-se coletas de amostras de solo (amostras compostas) na área próxima a nascente do córrego (dentro da área de estudo), local que apresenta fragmentos da vegetação original, considerado sem alterações antrópicas. 
Coleta de amostras de sedimentos fluviais em suspensão

As amostras de sedimentos fluviais em suspensão que foram utilizadas para as análises de $\mathrm{Co}, \mathrm{Cd}$ e As, foram coletadas durante os eventos de chuva na seção hidrossedimentométrica no período de 2003 a 2006, sendo realizadas várias coletas de amostras durante a onda de cheia (subida e descida). As amostragens foram realizadas em diferentes momentos dos eventos para contemplar os possíveis efeitos da variabilidade temporal das concentrações de metais traço decorrente das condições das vazões e das condições da bacia vertente.

As amostras de sedimentos em suspensão foram coletadas com o auxílio de um amostrador do tipo US DH-81 para estudos de qualidade dos sedimentos que é feito de Teflon ${ }^{\circledR}$. O material coletado foi transferido para galões de polipropileno de 20 litros. As amostras centrifugadas foram secas em estufa com temperatura máxima de $40^{\circ} \mathrm{C}$. As amostras secas foram transferidas para frascos de polipropileno de $50 \mathrm{~mL}$ e congeladas, evitando-se o contato com utensílios de metal para que as amostras não fossem contaminadas, conforme procedimento sugerido por Horowitz (1991), Mudroch e Azcue (1995), Mudroch, Azcue e Mudroch, (1997) e Poleto e Gonçalves (2006).

Todos os galões, baldes e vidrarias envolvidas no procedimento de coleta e concentração dos sedimentos para posterior congelamento foram lavados com água destilada, mergulhados em solução de ácido nítrico $14 \%$ (v/v) por 24 horas e depois novamente enxaguados com água deionizada.

\section{Digestão ácida total dos sedimentos}

Foram selecionados $\mathrm{Cd}$, Co e As como elementos de estudo, e estes tiveram suas concentrações totais determinadas por digestão ácida $\left(\mathrm{HCl}-\mathrm{HF}-\mathrm{HClO}_{4}-\right.$ $\mathrm{HNO}_{3}$ ) para destruição total dos minerais dos sedimentos. As amostras foram analisadas em duplicata mais um "branco".

Instrumentos, vidrarias, reagentes e soluções

As pipetas foram exclusivas para cada reagente. As vidrarias utilizadas foram lavadas em água destilada, ficando submersas em ácido nítrico $14 \%(\mathrm{v} / \mathrm{v})$ por 24 horas e depois são novamente enxaguadas com água deionizada (POLETO; GONÇALVES, 2006).

Os reagentes analíticos e as soluções extratoras que foram preparados para as análises são da marca Merck $^{\circledR}$. A água utilizada para as diluições é do tipo Milli-Q (extra-pura).

A determinação dos elementos selecionados para o estudo de poluentes na área de estudo foram analisados através dos extratos resultantes da digestão total dos sedimentos utilizados em cada fase da extração seqüencial, através de espectroscopia de emissão indutiva de plasma acoplado (ICP-OES), da marca Perkin Elmer, pelo Laboratório de Solos da Universidade Federal do Rio Grande do Sul. 


\section{RESULTADOS E DISCUSSÕES}

\section{Infra-estrutura urbana}

Por meio do levantamento referente à infra-estrutura urbana, constatou-se que $31 \%$ das ruas ou passagens de acesso presentes na área são pavimentadas (asfalto ou paralelepípedos) e $69 \%$ são não-pavimentadas ou de terra. As ruas não-pavimentadas foram consideradas como uma das mais importantes fontes potenciais de sedimentos disponíveis para serem carreados para dentro do córrego, pois se verificou nos levantamentos de campo a presença constante de sulcos de erosão especialmente nas ruas onde os terrenos apresentam declividades mais acentuadas.

O abastecimento de água potável atinge aproximadamente $100 \%$ da população, porém não existe sistema de captação de esgoto sanitário ou doméstico. Pode-se observar na Figura 1 os diferentes destinos que são expostos os dejetos domésticos (esgoto) da área de estudo.

O lançamento direto de esgoto no corpo d'água aparece como vetor de transmissão de doenças, e transforma a área de estudo em condição insalubre para a realização das coletas de amostras e estudos complementares. Este tipo de condição exigiu medidas preventivas para que os riscos de contaminação dos técnicos fossem minimizados, conforme sugerido por Merten e Poleto (2006).

Segundo Poleto et al. (2005), a maior parte dos esgotos lançados no corpo d'água é clandestina (31\%), ou seja, lançam o esgoto sanitário doméstico "in natura" para o córrego, $43 \%$ fizeram ligação direta com a canalização de drenagem de águas pluviais e o esgoto acaba chegando ao canal, 7\% das casas construíram fossas rudimentares (fossa negra), e os 19\% restantes construíram fossas rudimentares e as ligaram com a canalização de drenagem de águas pluviais para não realizar a devida manutenção (esgotamento sazonal), ou seja, o esgoto também vai para o corpo d'água depois de algum tempo. Essa condição constitui-se em uma das fontes potenciais de contaminação do sistema fluvial da bacia, pois podem estar aportando no corpo d'água além de matéria orgânica, agentes patogênicos, metais pesados e nutrientes. 


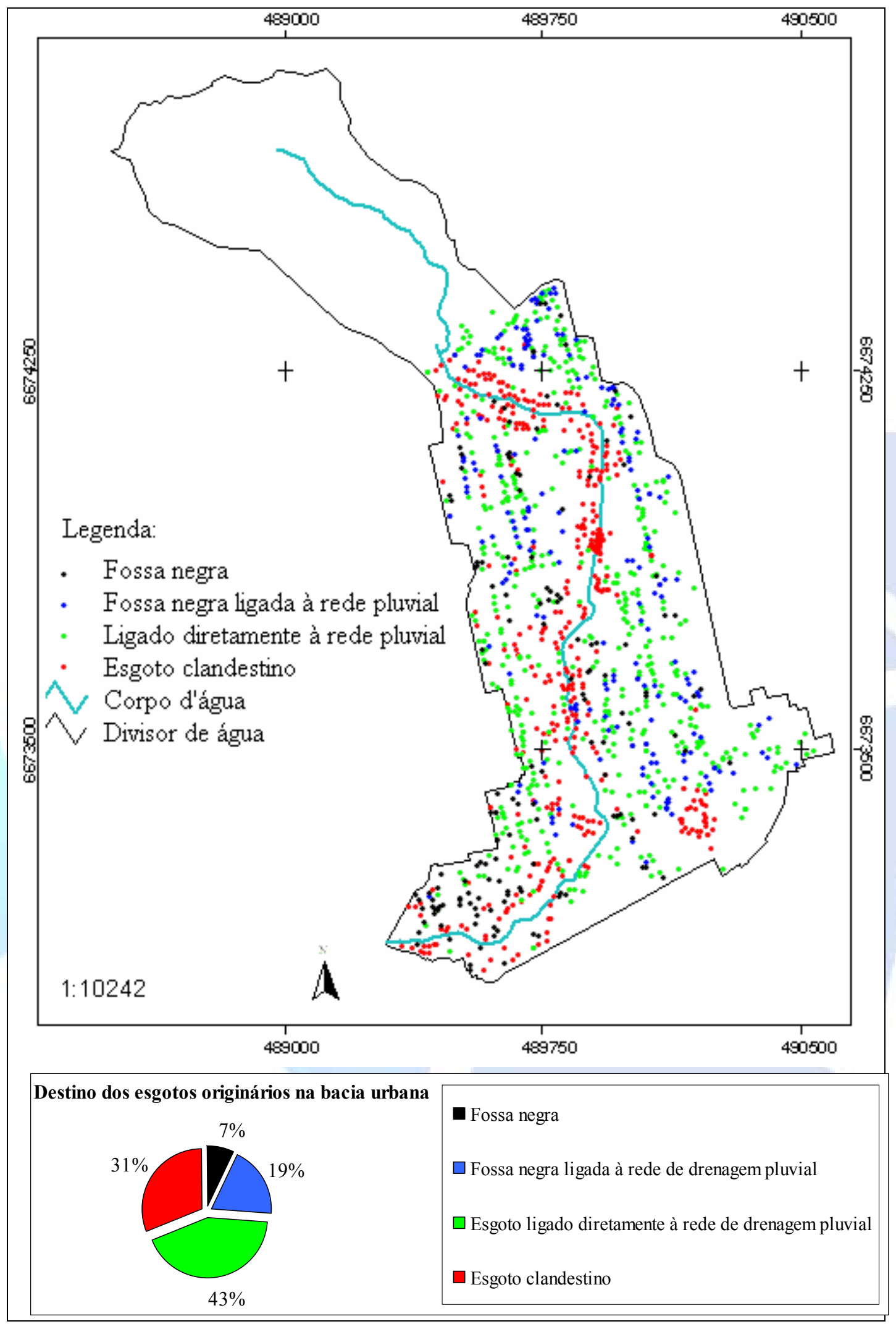

FIGURA 1. Saneamento básico da área de estudo. 
Análises das concentrações totais de $\mathrm{Co}, \mathrm{Cd}$ e As

$\mathrm{Na}$ Tabela 4, pode-se observar os valores médios das concentrações obtidas para os três elementos analisados, além da mediana, desvio padrão e valores de "background".

TABELA 4 - Concentrações totais médias, mediana, desvio padrão e background das 29 amostras de sedimentos fluviais em suspensão analisadas entre os anos de 2003 e 2006

\begin{tabular}{|c|c|c|c|c|c|}
\hline Elementos & $\begin{array}{c}\mathrm{n}^{\circ} \text { de amostras } \\
\text { analisadas }\end{array}$ & $\begin{array}{l}\text { Média } \\
\left(\mu g g^{-1}\right)\end{array}$ & $\begin{array}{c}\text { Mediana } \\
\left(\mu \mathrm{g} \mathrm{g}^{-1}\right)\end{array}$ & Desvio Padrão & $\begin{array}{c}\text { Background } \\
\left(\mu \mathrm{g} \mathrm{g}^{-1}\right)\end{array}$ \\
\hline $\mathrm{Cd}$ & 29 & 0,62 & 0,60 & 0,23 & 0,07 \\
\hline $\mathrm{Co}$ & 29 & 25,07 & 26,70 & 8,58 & 19,00 \\
\hline As & 29 & 59,88 & 72,00 & 26,03 & 55,00 \\
\hline
\end{tabular}

Para facilitar a visualização dos resultados, as concentrações das análises de cada amostra de sedimento fluvial em suspensão foram grafadas nas Figuras 2, 3 e 4, utilizando-se os valores de referência dos três Guidelines apresentados (OMEE, 1993; VROM, 2000; BRASIL, 2004) e os resultados de background local. Nas Figuras pode-se observar que há uma variação muito grande no que diz respeito aos valores de cada Guideline em relação aos valores de background local, refletindo as diferentes condições na qual estes foram elaborados.

$\mathrm{Na}$ Figura 2 que apresenta os resultados obtidos nas análises de As, pode-se observar a importância dos valores de background local, uma vez que este se encontra muito acima dos valores sugeridos por todos os Guidelines, igualando-se ao valor considerado de intervenção pelo VROM (2000). Essa concentração elevada para o background pode também estar indicando a contaminação da área vegetada por esse elemento, provavelmente pela atmosfera. A concentração média das amostras de sedimentos foi de $59,88 \mu \mathrm{g} \mathrm{g}^{-1}$, mediana de $72,00 \mu \mathrm{g} \mathrm{g}^{-1}$, valor máximo de $93,00 \mu \mathrm{g} \mathrm{g}^{-1}$, mínimo de $12,00 \mu \mathrm{g} \mathrm{g}^{-1}$ e desvio padrão de 26 .

Apesar disso, as concentrações de As estariam muito acima do que seria considerado normal para a área, o que pode indicar o seu enriquecimento devido a ações antrópicas. Em estudos realizados por De Carlo, Beltran e Tomlinson, (2004) em uma pequena área urbanizada do Hawaii, as médias das amostras de sedimentos coletadas durante eventos de chuva, em dois canais locais, apresentaram médias de 27 e $66 \mu \mathrm{g} \mathrm{g}^{-1}$, sendo que essas concentrações foram consideradas elevadas pelos pesquisadores e portanto resultantes da influência antrópica local. Apenas em estudos que mostraram a contaminação de locais afetados por minas, como foi apresentado por Bird et al. (2003) em seus estudos na Romênia e Hungria, as concentrações de As chegaram a picos de $240 \mu \mathrm{g} \mathrm{g}^{-1}$. 


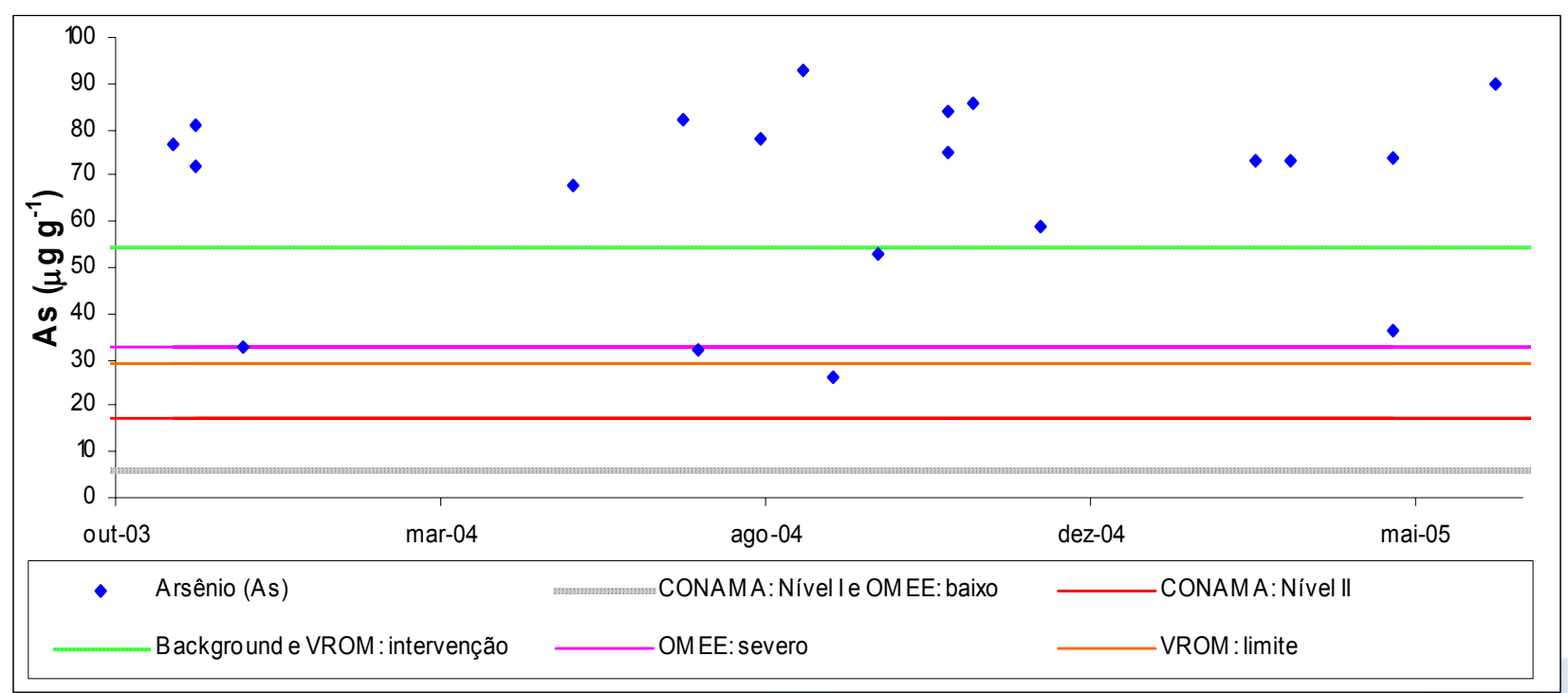

FIGURA 2. Concentração total de $\mathrm{As}\left(\mu \mathrm{g} \mathrm{g}^{-1}\right)$ em 29 amostras de sedimentos fluviais em suspensão coletadas entre 2003 e 2006, e os valores de referência dos "Guidelines" e do "background" local.

A Figura 3 apresenta os valores obtidos para o $\mathrm{Cd}$, e pode-se observar que a maior parte das amostras ficaram abaixo de todos os limites estabelecidos pelos três Guidelines, e que os limites máximos de cada um divergiram muito. As concentrações das amostras apresentaram uma média de $0,62 \mu \mathrm{g} \mathrm{g}^{-1}$, mediana de 0,60 $\mu \mathrm{g} \mathrm{g}^{-1}$, concentração máxima de $1,60 \mu \mathrm{g} \mathrm{g}^{-1}$, mínima de $0,30 \mu \mathrm{g} \mathrm{g}^{-1}$ e desvio padrão de 0,23 , e portanto todos os resultados ficaram entre 2 e 4 vezes maiores que os valores encontrados para o background, sugerindo a entrada desse metal no sistema. Mesmo assim, os resultados podem ser considerados similares a outros estudos, como por exemplo os realizados no rio Rhône que ficaram entre 0,3 e $4,2 \mu \mathrm{g} \mathrm{g}^{-1}$ (SANTIAGO, THOMAS E LARBRAIGT., 1993).

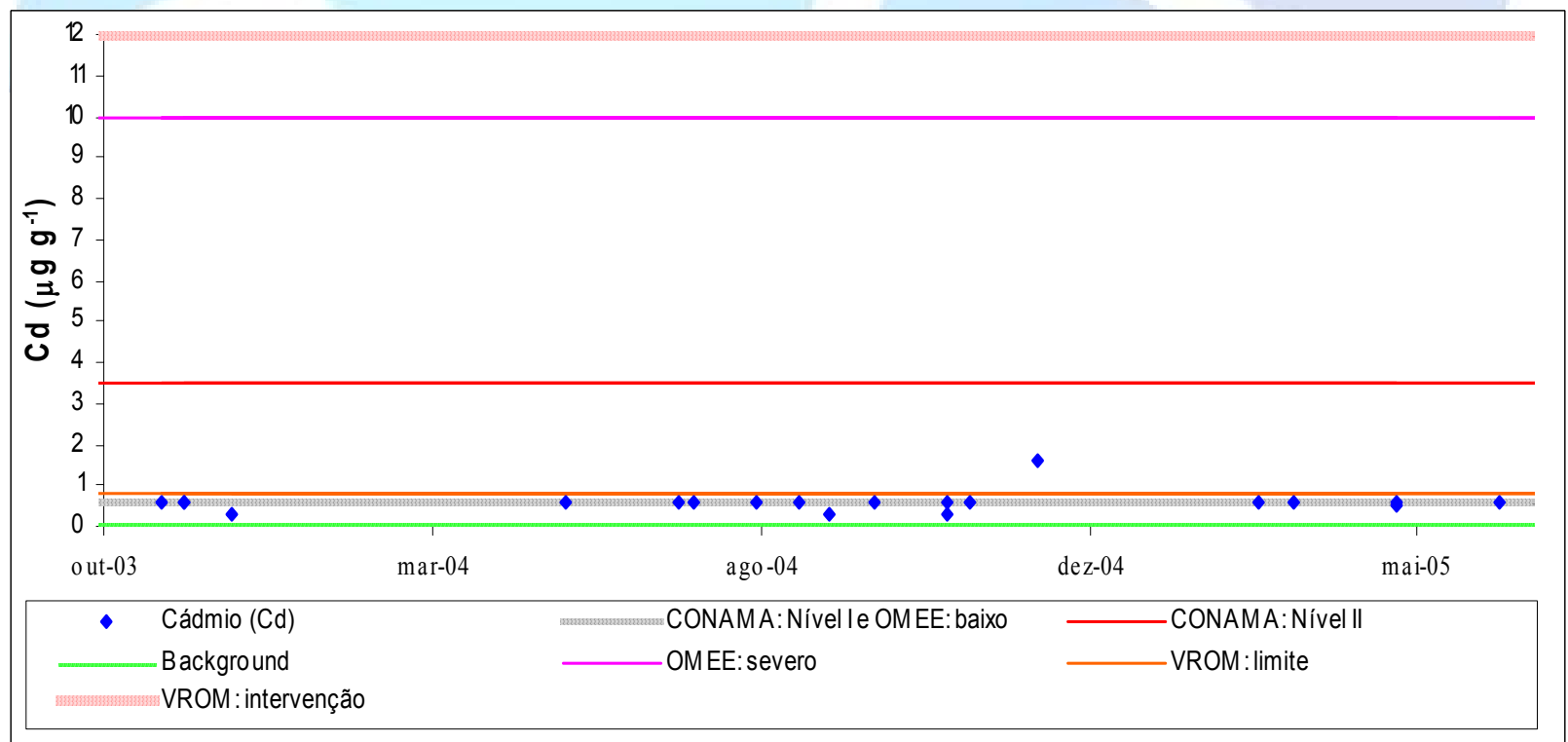

FIGURA 3. Concentração total de $\mathrm{Cd}\left(\mu \mathrm{g} \mathrm{g}^{-1}\right)$ em 29 amostras de sedimentos fluviais em suspensão coletadas entre 2003 e 2006, e os valores de referência dos Guidelines e do background local. 
As concentrações totais de cobalto encontradas durantes as análises são mostradas na Figura 4, obtendo-se uma média de $25,07 \mu \mathrm{g} \mathrm{g}^{-1}$, mediana de $26,70 \mu \mathrm{g}$ $\mathrm{g}^{-1}$, máximo de $41,70 \mu \mathrm{g} \mathrm{g}^{-1}$, mínimo de $8,50 \mu \mathrm{g} \mathrm{g}^{-1}$ e desvio de 8,58. Assim como ocorreu com o background de referência para o arsênio, o valor encontrado como referência local (background) para o Co ficou acima da recomendação do limite proposto pelo VROM (2000) que foi obtido em estudos de regiões holandesas. De qualquer forma, os resultados ficaram muito abaixo do limite considerado de intervenção e severo pelos Guidelines, e apesar de superiores, a maior parte das concentrações não chegaram a ser duas vezes maior que os valores de background.

Portanto, levando-se principalmente em consideração que por se tratar de uma bacia urbana residencial, não-industrial, conforme previamente apresentado na caracterização da área de estudo, a mesma não possui industrias que possam estar participando do processo de enriquecimento de metais nos sedimentos fluviais em suspensão, o que torna os esgotos sem tratamento, conforme a própria literatura apresentada ao longo do trabalho (MIYAZAWA, 1996; GUÉGUEN et al., 2000; GROMAIRE et al., 2001; PARDOS et al., 2004; BROWN e PEAKE, 2006), uma fonte importante para o aumento de metais sorvidos em sedimentos fluviais originários de bacias urbanas.

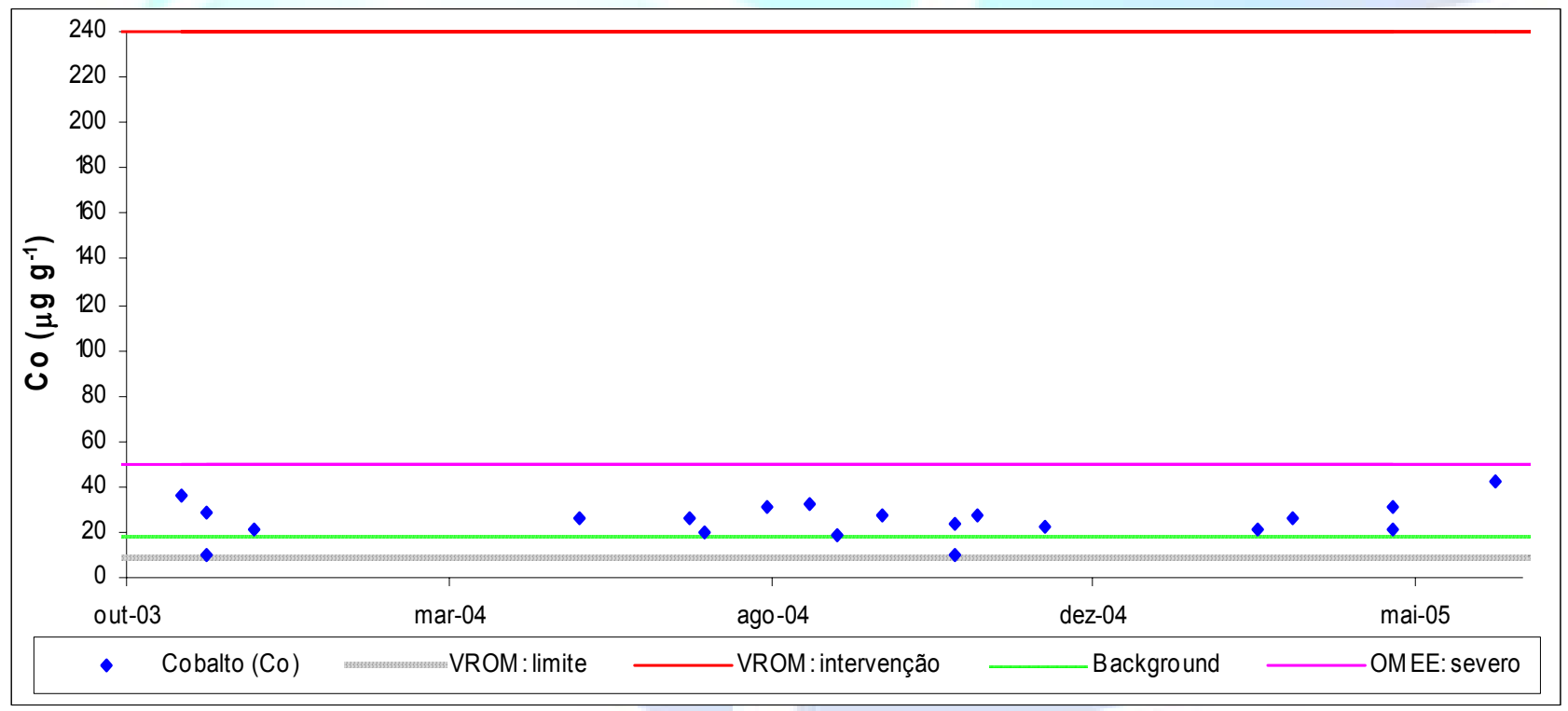

FIGURA 4. Concentração total de Co $\left(\mu \mathrm{g} \mathrm{g}^{-1}\right)$ em 29 amostras de sedimentos fluviais em suspensão coletadas entre 2003 e 2006, e os valores de referência dos Guidelines e do background local.

Outro fator que pode explicar o aumento dos poluentes encontrados nas análises dos sedimentos fluviais em suspensão é a grande contribuição relativa de sedimentos das ruas pavimentadas. As amostras de sedimentos que foram coletadas nas ruas pavimentadas apresentaram elevadas concentrações desses elementos (Tabela 5). Esse resultado está em conformidade com os diversos trabalhos citados (HOPKE et al., 1980; FERGUSSON e KIM, 1991; DE MIGUEL et al., 1997; LI et 
al., 2001; BANERJEE, 2003; JAFFÉ et al., 2003; CHARLESWORTH et al., 2003; ADACHI e TAINOSHO, 2005; TAYLOR, 2007) que apresentam as ruas pavimentadas em ambientes urbanos como a maior fonte de metais e outros contaminantes.

TABELA 5 - Concentrações totais médias dos elementos analisados nas amostras de sedimentos das ruas pavimentadas.

\begin{tabular}{cccc}
\hline Fonte & \multicolumn{3}{c}{ Elementos (Concentrações médias em $\left.\boldsymbol{\mu g} \mathbf{g}^{\mathbf{- 1}}\right)$} \\
\cline { 2 - 4 } & $\mathbf{A s}$ & $\mathbf{C o}$ & $\mathbf{C d}$ \\
\hline Ruas Pavimentadas & 52,07 & 24,05 & 0,45 \\
\hline
\end{tabular}

\section{CONCLUSÕES}

A falta de infra-estrutura urbana permite concluir que essa área é bastante representativa para a maioria das áreas urbanas residenciais encontradas na periferia das grandes cidades brasileira e os esgotos lançados diretamente no corpo d'água é uma fonte importante de contaminantes.

A comparação dos valores das concentrações totais dos elementos obtidos dos sedimentos fluviais em suspensão em relação aos valores de referência estabelecidos nos Guidelines se mostrou limitada ou inadequada para possibilitar um enquadramento das concentrações obtidas neste trabalho. Essa condição sugere a necessidade de utilização dos valores de referência local (background) para estudos de enriquecimento dos sedimentos por contaminantes de origem antrópica.

Os valores de concentração total dos elementos analisados (Cd, Co e As) apresentaram concentrações acima dos padrões utilizados como background local. Para o $\mathrm{Cd}$, os valores de concentração total foram em média três vezes maiores que os valores de base ou background. Essa condição caracteriza de forma bastante evidente a contribuição da ação antrópica (baterias, resíduos de oficinas e pequenas fábricas) no processo de enriquecimento de metais nos sedimentos urbanos.

\section{REFERÊNCIA}

ADACHI, K.; TAINOSHO, Y. Single particle characterization of size-fractionated road sediments. Applied Geochemistry, v.20, n.5, p.849-859, 2005.

BAIRD, C. Química ambiental. 2.ed. Porto Alegre: Bookman, 2002. 622p.

BANERJEE, A. D. K. Heavy metal levels and solid phase speciation in street dusts of Delhi, India. Environmental Pollution, v.123, p.95-105, 2003. 
BIRD, G.; BREWER P.A.; MACKLIN, M.G.; BALTEANU, D.; DRIGA, B.; SERBAN, M.; ZAHARIA, S.. The solid state partitioning of contaminant metals and As in river channel sediments of the mining affected Tisa drainage basin, Northwestern Romania and Eastern Hungary. Applied Geochemistry, v.18, p.1583$1595,2003$.

BROWN, J. N.; PEAKE, B. M. Sources of heavy metals and polycyclic aromatic hydrocarbons in urban stormwater runoff. Science of the Total Environment, v.359, p.145-155, 2006.

BUYKX, S. E. J.; BLEIJENBERG, M.; HOOP, M.A.G.T.V.D; LOCH, J. P. G.. The effect of oxidation and acidification on the speciation of heavy metals in sulfide-rich freshwater sediments using a sequential extraction procedure. Journal

Environmental Monitoring, v.2, p.23-27, 2000.

DOI: $\underline{10.1039 / \mathrm{a} 907790 \mathrm{a}}$

CCME - CANADIAN COUNCIL OF MINISTERS OF THE ENVIRONMENT. Protocol for the derivation of Canadian sediment quality Guidelines for the protection of aquatic life. Ottawa: Environment Canada, Guidelines Division Technical Secretariat of the CCME Task Group on Water Quality Guidelines. EPC98E, 1995.

CHARLESWORTH, S. M.; EVERETT, M.; McCARTHY, R.; ORDÓÑEZ, A; DE MIGUEL, E. A comparative study of heavy metal concentration and distribution in deposited street dusts in a large and a small urban area: Birmingham and Conventry, West Midlands, UK. Environment International, v.29, p.563-573, 2003.

BRASIL. MINISTÉRIO DO MEIO AMBIENTE. Conselho Nacional do Meio Ambiente - CONAMA. Resolução CONAMA, no 344, de 25 de março de 2004. Disponível em: $<$ http://www.mma.gov.br/port/conama $>$. Acesso em: 06 mar. 2007.

DE CARLO, E. H.; BELTRAN, V. L.; TOMLINSON, M. S. Composition of water and suspended sediment in streams of urbanized subtropical watersheds in Hawaii. Applied Geochemistry, v.19, p.1011-1037, 2004.

DELETIC, A. Modelling of water and sediment transport over grassed areas. Journal of Hydrology, v.248, p.168-182, 2001.

DE MIGUEL, E.; LLAMAS, J.F.; CHACON, E.; BERG, T.; LARSSEN, S.; ROYSET, O.; VADSET, M. Origin and patterns of distribution of trace elements in street dust: Unleaded petrol and urban lead. Atmosphery Environment, v.31, p.2733-2740, 1997. 
FERGUSSON, J. E.; KIM, N. D. Trace elements in street and house dusts: Sources and speciation. Science of Total Environment, v.100, p.125-150, 1991.

FOSTER, I. D. L.; CHARLESWORTH, S. M. Heavy metals in the hydrological cycle: Trends and explanation. Hydrological Processes, v.10, p.227-261, 1996.

GROMAIRE,M.C.; GARNAUD,S.; SAAD, M.; CHEBBO, G. Contribution of different sources to the pollution of wet weather flows in combined sewers. Water Researches, v.35, n.2, p.521-533, 2001.

GUÉGUEN, C.; DOMINIK, J., PARDOS, M., BENNINGHOFF, C.; THOMAS, R. L. Partition of metals in the Vistula river and in effluents from sewage treatment plants in the region of Cracow (Poland). Lakes \& Reservoirs: Research and Management, v.5, p.59-66, 2000.

HOPKE, P. K.; LAMB, R. E.; NATUSCH, D. F. S. Multielemental characterization of urban roadway dust. Environment Science Technology, v.14, p.164-172, 1980.

HOROWITZ, A. J. A primer on sediment-trace element chemistry. 2.ed. Chelsea, EUA: Lewis Publishers, 1991. 136p.

HOROWITZ, A. J. The use of suspended sediment and associated trace elements in water quality studies. International Association of Hydrological Sciences: IAHS Special Publication, 1995. 58p.

HURSTHOUSE, A. S. The relevance of speciation in the remediation of soils and sediments contaminated by metallic elements-an overview with specific examples from central Scotland. Journal Environmental Monitoring, v.3, p.49-60, 2001.

JAFFÉ, R.;GARDINALI, P. R.; CAI, Y.; SUDBURRY, A.; FERNANDEZ, A.; HAY, B.J. Organic compounds and trace metals of anthropogenic origin in sediments from Montego Bay, Jamaica: Assessment of sources and distribution pathways. Environmental Pollution, v.123, p.291-299, 2003.

LEE, P.K.; YU Y.H.; YUN S.T.; MAYER, B. Metal contamination and solid phase partitioning of metals in urban roadside sediments. Chemosphere, v.60, n.5, p.672$689,2005$.

LI, X. D.; POON, C. S.; LIU, P. S. Heavy metal contamination of urban soils and street dusts in Hong Kong. Applied Geochemistry, v.16, p.1361-1368, 2001.

LIJZEN, J. P. A.; BAARS, A.J; OTTE, P.F.; RIKKEN, M.G.J.; SWARTJES, 
F.A.;VERBRUGGEN, E.M.J.; VAN WEZEL, A.P. Technical evaluation of the intervention values for soil/sediment and groundwater - Human and ecotoxicological risk assessment and derivation of risk limits for soil, aquatic sediment and groundwater. Holanda: RIVM - National Institute of Public Health and the Environment, 2001. Report 711701023. 147p.

MACKLIN, M. G.; BREWER, P.A.; BALTEANU, D.; COULTHARD, T.J.; DRIGA, B.; HOWARD, A. J.; ZAHARIA, S. The long term fate and environmental significance of contaminant metals Rreleased by the January and March 2000 mining tailings dam failures in Maramures county, upper Tisa basin, Romania. Applied Geochemistry, v.18, p.241-257, 2003.

MACKLIN, M. G.; KLIMEK, K. Dispersal, storage and transformation of metal contaminated alluvium in the upper Vistula basin, southwest Poland. Applied Geochemistry, v.12, p.7-30, 1992.

MARCHAND, C.; LALLIER-VERGES, E.; BALTZER, F.; ALBERIC, P.; COSSA, D.;BAILLIF, P. Heavy metals distribution in mangrove sediments along the mobile coastline of French Guiana. Marine Chemistry, v.98, p.1-17, 2006.

MERTEN, G. H.; POLETO, C. Rede de monitoramento e coleta de amostras. In: Poleto, C.; Merten, G. H. (Orgs.). Qualidade dos sedimentos. Porto Alegre: ABRH, 2006. p.1-38.

METRE, P. C. V.; MAHLER, B. J. The contribution of particles washed from rooftops to contaminant loading to urban streams. Chemosphere, v.52, p.1727-1741, 2003.

MIYAZAWA, M. Estado da arte: metais pesados no solo e na planta - Projeto interdisciplinar para a definição de critérios sanitários agronômicos e ambientais para implantação da reciclagem agrícola do lodo de esgoto. Paraná: Instituto Ambiental do Paraná, 1996.

MUDROCH, A.; AZCUE, J. Manual of aquatic sediment sampling. Florida, EUA: CRC Press, 1995. 219p.

MUDROCH, A.; AZCUE, J.; MUDROCH, P. Manual of physico-chemical analysis of aquatic sediments. Florida, EUA: CRC Press, 1997. 287p.

OMEE - ONTARIO MINISTRY OF THE ENVIRONMENT AND ENERGY. Guidelines for the protection and management of aquatic sediment quality in Ontario. Toronto: Ontario Ministry of the Environment and Energy, 1993. 
PACKMAN, J. J.; COMINGS. K. J.; BOOTH, D. B. Using turbidity to determine total suspended solids in urbanizing streams in the puget lowlands. In: Confronting Uncertainty: Managing Change In Water Resources And The Environment, 1999. Canadian Water Resources Association annual meeting. Vancouver. p.158-165.

PARDOS, M.; BENNINGHOFF, C.; ALENCASTRO, L. F., WILDI, W. The impact of a sewage treatment plant's effluent on sediment quality in a small bay in Lake Geneva (Switzerland-France). Lakes \& Reservoirs: Research and Management, v.9, p.41-52, 2004.

PATKOWSKA, J. M.; HURSTHOUSE, A.; KIJ, H. P. The interaction of heavy metals with urban soils: sorption behaviour of $\mathrm{Cd}, \mathrm{Cu}, \mathrm{Cr}, \mathrm{Pb}$ and $\mathrm{Zn}$ with a typical mixed brownfield deposit. Environment International, v.31, p.513-521, 2005.

POLETO, C.; MERTEN, G. H.; SILVEIRA, A. L. L. Socio-economic impacts on fluvial system an urban watershed in southern Brazil. In: $10^{\text {th }}$ INTERNATIONAL CONFERENCE ON URBAN DRAINAGE, 2005, Copenhagen, Dinamarca. X ICUD. Copenhagen: University of Denmark, 2005.

POLETO, C.; GONÇALVES, G. R. Qualidade das amostras e valores de referência. In: Poleto, C.; Merten, G. H. (Orgs.). Qualidade dos sedimentos. Porto Alegre: ABRH, 2006. p.237-277.

PORTO, M. F. A. Aspectos qualitativos do escoamento superficial em áreas urbanas. In: Tucci, C. E. M.; Porto, R. L.; Barros, M. T. (orgs). Drenagem urbana. Porto Alegre: ABRH/Editora da Universidade/UFRGS, 1995. p.387-428.

RUSSELL, M. A.; WALLING, D. E.; HODGKINSON, R. A. Suspended sediment sources in two small lowland agricultural cachtments in the UK. Journal of Hydrology, v.252, p.1-24, 2001.

SÁNCHEZ-RODAS D.; GÓMEZ-ARIZA, J.L.; GIRÁLDEZ, I.;. VELASCO, A.; MORALES, E. Arsenic speciation in river and estuarine waters from southwest Spain. Science of the Total Environment, v.345, p.207-217, 2005.

SANTIAGO, S.; THOMAS, R. L.; LARBRAIGT, G. Comparative ecotoxicology of suspended sediment in the lower Rhône river using algal fractionation, Microtox ${ }^{\circledR}$ and Daphnia Magna bioassays. Hydrobiologia, v.252. p.231-244, 1993.

SINGH, K. P.; MOHAN, D.; SINGH, V.K.; MALIK, S. Studies on distribution and fractionation of heavy metals in Gomti river sediments - a tributary of the Ganges, India. Journal of Hydrology, v.312, p.14-27, 2005. 
TAYLOR, K. Urban environments. In: Perry, C.; Taylor, K. (Eds.). Environmental sedimentology. UK: Blackwell Publishing Ltda., 2007. 441p.

TAYLOR, S. E.; BIRCH, G. F.; LINKS, F. Historical catchment changes and temporal impact on sediment of the receiving basin, Port Jackson, New South Wales. Australian Journal of Earth Sciences, v.51, p.233-246, 2004.

VROM - MINISTERY OF HOUSING SPATIAL PLANNING AND ENVIRONMENT. Circular on target values and intervention values for soils remediation. Netherlands Government Gazette, v.39, p.1-11, 2000. 